Historic, Archive Document

Do not assume content reflects current scientific knowledge, policies, or practices. 



\section{FALL PRICE LIST, 1932}

II

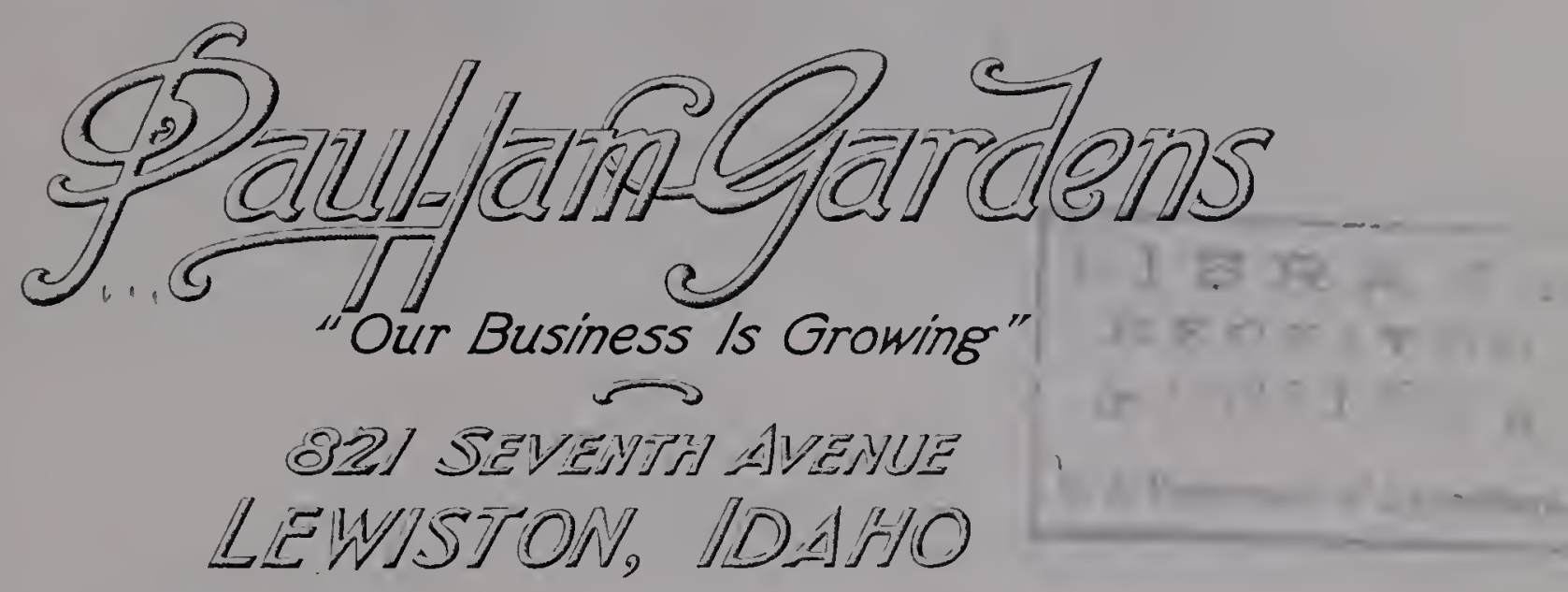

\section{TERMS OF SALE}

All prices made are for cash. However, we will hold goods until March 1st, 1933, if $25 \%$ is paid down to hold order. Prices here hold until issuance of spring list in. January, 1933. All stock is offered subject to prior sale.

We never substitute varieties without your permission. If you can use substitutes, rlease note. In case size is sold out. we will substitute next size unless forbiden, giving at least equal value.

No item filled for less than $20 \mathrm{c}$. If list states 10 bulblets for 100 we will fill order for that amount. If it states, for instance, 100 bulblets for $90 \mathrm{c}$, please order at least $20 \mathrm{c}$ worth. No order accepted for less than $\$ 1$.

All prices in this catalog include packing and transportation to any place in the Inited States or Canada. Foreign countries please add $15 \%$ for postage. We ship to Dec. 10 th in the fall and after March 1st in the spring at our own risk. If you wish your bulbs during the winter you assume the possibility of their loss by freezing. We reserve the right to ship by either mail or express.

On account of the present fluctuation of foreign exchange, we must ask customers in foreign countries to remit by international money order or United States exchange. Foreign currency will be credited only at exchange rate current when received.

\section{DISCOUNTS}

A discount of $5 \%$ will be given on all orders before December 1,1932 .

A discount of $15 \%$ is offered to customers who will pay transportation charges. In this case, parcels post shipments will be sent C. O. D. for charges.

Quantity discounts will be given as follows: $5 \%$ on $\$ 10$ or over; $10 \%$ on $\$ 25$ orders; $15 \%$ on $\$ 100$ orders.

Above discounts apply to cash orders only. They do not apply to collections or to the following varieties: Acadia, Andreas Hofer, Blue Admiral, Coronation, Debonair, Golden Cup, Hercules, Mrs. T. E. Langford, Newington, Noel I. Reeve, Red Phipps, Rideau, Rapture, Smiling Maestro, Valencia and Wasaga.

\section{WE PROTECT YOU}

We will sell you an order of bulbs at as low a price as any other grower's list gives you. If after you make out your order, you find the prices of some other grower are lower for the total amount, remit the amount other grower's list calls for, mentioning $h$ is name.

If after you get our spring catalog, you find that you can buy an order sent us in the fall at a cheaper price from spring list, apprise us of the fact and we will send extra bulbs to make up for any deficiency. 


\section{PLEASE READ THIS CAREFULLY}

In getting out this list, we have tried to economize on space, shortening descriptions and listing each variety in just one quantity. To do this we hare figured out the' following system.

Where listed by 1000 only, divide by 8 to get the 100 price. If listed by 100 only, divide by 8 to get the 10 price. Where the 10 price only is given divide by 9 for the each price.

The above may take a little figuring but we believe this system is equitable to all. As is usual, fire or more will be sold at 10 price. 25 or more at 100 price and 250 at 1000 price. On many kinds we can supply bulbs in thousands and bulblets by the quart. Wrrite us.

All bulbs guaranteed true-to-llame and frce from disease. We have bcen unable to find any trace of sladiolus thrips in our gardens so believe we have as yet escaped this pest.

\section{Varieties and Prices}

\begin{tabular}{|c|c|c|c|c|c|c|}
\hline & \multirow[b]{2}{*}{ Per } & \multirow{2}{*}{$\begin{array}{r}\text { Large } \\
11 / 4 \text { "up }\end{array}$} & \multirow{2}{*}{$\begin{array}{r}\text { Med. } \\
3 / 4 \text { " up }\end{array}$} & \multirow{2}{*}{$\begin{array}{c}\text { Small } \\
3 / 8 \text { "up }\end{array}$} & \multicolumn{2}{|c|}{ Bulblets. } \\
\hline & & & & & & \\
\hline ACADIA-Geranium pink, with yellow throat & 1 & 1.00 & & & 10 & .50 \\
\hline A. E. KUNDERD-Large salmon rose, blotched & 100 & 4.00 & 2.00 & 1.00 & 1000 & 1.00 \\
\hline AFLAME-Immense begonia rose, shaded flame & 100 & 4.00 & 2.25 & 1.00 & 1000 & 1.00 \\
\hline A. G. BLIGHT-Very dark l'ed, shaded black & 10 & .60 & .30 & .15 & 1000 & 2.00 \\
\hline A. H. WOODFULL-Strong growing lavender & 10 & 13.50 & 7.20 & & 100 & 6.00 \\
\hline AIDA - Large early dark blue. Extra fine .... & 100 & 7.20 & 3.60 & 2 & 1000 & 4.00 \\
\hline ALASKAN SUNSET-Coppery pink, overcast sray & 10 & 4.50 & 2.25 & 1.35 & 1000 & 8.00 \\
\hline 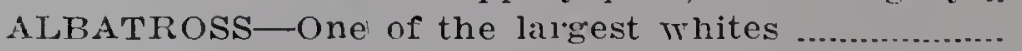 & 10 & 2.00 & 1.25 & .70 & 1000 & 10.00 \\
\hline ALLAWAH-Very tall blue. Very distinct & 10 & 2.00 & 1.00 & .60 & 100 & 1.00 \\
\hline AMAZON-Exhibition lavender pink, white $t$ & 1 & 2.00 & & .75 & 1 & .25 \\
\hline AMBROSIA-Old rose, prominent blotch ....... & 1 & .40 & .25 & & 10 & .25 \\
\hline AMIETHYST-Exhibition spike & 1 & .50 & & .25 & & \\
\hline ANDREAS HOFER-New exhibition salin & 1 & 8.00 & & & 1 & 1.00 \\
\hline ANGEL'S DREAM-Coral pink with go & 10 & 1.15 & .60 & .30 & & 2.00 \\
\hline ANNIE LAUR & 100 & 3.00 & 1.50 & .7 & 1000 & .60 \\
\hline APPLE BLOSSOM (Hol.)-Fine, tall, light pink & 10 & .75 & & .25 & 100 & .15 \\
\hline APRICOT GLOTV-Clear apricot, & 100 & 3.00 & 1.50 & .80 & 1000 & .80 \\
\hline AVE MARIA-Tall light blue, good gror & 10 & 1.00 & .50 & & 100 & .45 \\
\hline T & 10 & & 7.20 & 6 & 100 & .00 \\
\hline BARBARA PI & 1 & 3.00 & & & & \\
\hline BEAT ALL-Tall light rose pink, deeper feat & 1 & .50 & & .20 & & \\
\hline BELINDE-Large cream yellow. Distinctive & 100 & 3.60 & 2.00 & 1.00 & 1000 & 1.00 \\
\hline BENGAL BEAUTY-Striking brown and gold & 1 & .15 & .10 & & 10 & .10 \\
\hline BENTLEIGH-Exhibition white with cream throat & 1 & 1.00 & & & 1 & .15 \\
\hline TY SNOT-On & 100 & 4.00 & 2.00 & 1.00 & 1000 & 1.00 \\
\hline BETTY NUTHALL-Coral pink. Very out & 100 & 5.00 & 3.00 & 1.50 & 1000 & 1.00 \\
\hline ring brown orange with $\mathrm{m}$ & 1 & 1.50 & 1.00 & .50 & 10 & 1.35 \\
\hline BILI, SOWDEN-New crims & 10 & 6.75 & 4.50 & 2.2 & 100 & 5.00 \\
\hline BIRD OF PARADI & 1 & 2.00 & 1.00 & , & 1 & .10 \\
\hline NN-Larg & 1 & 1.00 & .60 & .35 & 10 & .90 \\
\hline BLUE ADMIRAI-Very fine new violet blue & 1 & 8.00 & & & 1 & .75 \\
\hline BLUE DANUBE-Amethyst blue, dark blotch & 10 & 9.00 & 5.40 & 3.15 & 100 & 4.00 \\
\hline FLUE MOON-New violet blue, very early & 100 & 28.80 & 14.40 & 7.20 & 1000 & 8.00 \\
\hline BLUE ROYALF-Deep violet blue, sereral open ... & 1 & & & & 1 & .40 \\
\hline BLUE TRIUMPHATOR-Large li & 10 & 13.50 & 7.20 & 4.50 & 100 & 8.00 \\
\hline BRONZE MIAID-Deep bronze, purple throat ..... & 1 & .40 & .25 & .15 & 10 & .25 \\
\hline$A-$ & 1 & 1.00 & & .30 & 1 & .10 \\
\hline CAMELOT-New coral pink with scarlet $t$ & 1 & 1.00 & & & 1 & .15 \\
\hline CANBERRA-Fine yellow. Strong and tall & 100 & 15.00 & 7.50 & 4.00 & 1000 & 4.00 \\
\hline CAPT. COURAGEOUS-Large, brinht mottled red.. & 1 & .25 & .15 & .10 & 10 & .15 \\
\hline CARA MIA—Tall, rose pink, purple feather ........... & 10 & .70 & .40 & .20 & 1000 & 1.50 \\
\hline CATMELITA-Large flower. Pink overlaid smoky & 10 & .50 & .30 & .15 & 100 & .15 \\
\hline CAROLUS CLUSIUS-Salmon pink, cream bloteh... & 100 & 7.20 & 3.60 & 1.80 & 1000 & 2.00 \\
\hline CARRICK-Very tall smoky pink ........................... & 10 & .90 & ...... & .25 & 100 & .25 \\
\hline CASSANDRA-Light rose pink, large scillet bl & 1 & 1.00 & .60 & .40 & 10 & .90 \\
\hline
\end{tabular}

One at one-ninth 10 price; 10 at one-eighth 100 price; 100 at one-eighth 1000 price 
CATHERINE COLEMAN-Large blotehed pink

\section{0}

CENTENAIRE-New bright cerise red

CHARMIEUSE-Bright scarlet, prominent blotch...

CHAS. BERTHIER-Very good tall lilac

CHAS. DICIENS-Bricht radiant purple Tall 100

CHAS. LINDBERGH-Fine pure pink, extra .......

CHICAGO-Very hearily ruffled red

\begin{abstract}
CHOCOLATE QUEEN-Fxtra sood chocolate brown
\end{abstract}
COMMANDER KOEHL_Extra good dark red.

COMRADE-Early slaty salmon, white throat...

CORANGAMITE-Bricht salmon rose, many out

CORONADO-Large white, purple throat ..............

CORONATION-Good spike of salmon rose ...........

CORYPHEE-Tall spikes, very lovely pure pink .... 10

DAMASCUS-Argyle purple and hellebore red

DAME MASSEY-Large bright rose, yellow throat

DAVID-New strons-growing cherry red

DAVID AIRDRIE-Carmine with cream throat.... 10

DAVID FISHER-Rare rose pink, cream throat ....

DEARBORN-Large salmon orange, tulip shape...

DEBONAIR-New salmon pink prim. grand. ........

DELICIOUS-White, edged shell pink.....................

DIABLO-Early orange red. Very tall ...................

DIGNITY-Pure rose pink, lighter throat ...............

DIRECTOR-Deep lavender, prominent blotch ........

DORA GILL-Very fine geranium pink, white throat

DOROTHY VERNON-Good white, yellow throat... 10

URACOCEPHALUS-Dragon's Head species ..........

DRAMA-Deep rose purple, darker throat ............ 1

DR. F. E. BENNETT-Most popular orange red ... 100

DR. MAYO-Lavender. Clear, distinctive color ....... 1

DR. MOODY - Early light lavender. Many open ... 100

DR. NELSON SHOOK-Very strong deep rose ..... 100

DUCHESS OF ATHOLL-Cherry red, white blotch 1

DUCHESS OF YORK (M) - Slaty rose, white throat 100

EMILE AUBRUN-Large bronze, cherry blotch ... 100

ENSEMBLE-Distinctly luffled, laciniated yellow... 10

ENSIGN-Large rosy red. White blotch.

E. O. BOASE-Exhibition white, tinted lavender ....

FSME DESAILLY - Very fine light geranium pink

FTENDARD-White, large red blotches

EUGLNIE-Deep rose pink, cream blotch

c.............

1

EULA TERRI-Early rose pink, lighter throat

EVE-Tery tall buff, suffused pink

FANNY CROSBY - Cream colored. Many blooms .

FACST-Salmon pink, overcast slate

FIELD MARSHAL-Large brown red marked slate

FOREST FIRE-Dark red flamed scarlet

FRANK II SHICK-Tall coral pink. Buff throat 100

FREDERICK CHRIST-Creamy La France pink.... 1

GALLANT LEADER-Strong grower. Rose pink... 10

GAY HUSSAR-Fine spike of salmon orange

100

.55

.50

2.00

2.00

.20

3.60

1.00

7.20

.90

16.50

.25

3.00

\subsection{5}

4.00

9.00

2.25

1.00

......

7.00

15.00

1.00

4.00

.50

.10

.35

.40

2.00

.50

.30

.35

3.00

.30

3.60

3.20

.50

6.00

$\begin{array}{ll}.30 & .15 \\ .20\end{array}$

$\begin{array}{ll}.25 & .20 \\ & .75\end{array}$

$\ldots \ldots . \quad \ldots \ldots$

..... .10

1.80

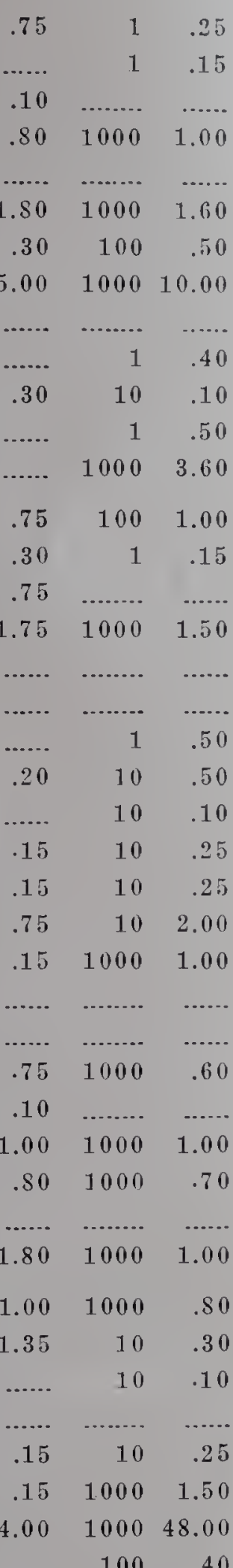

3.60

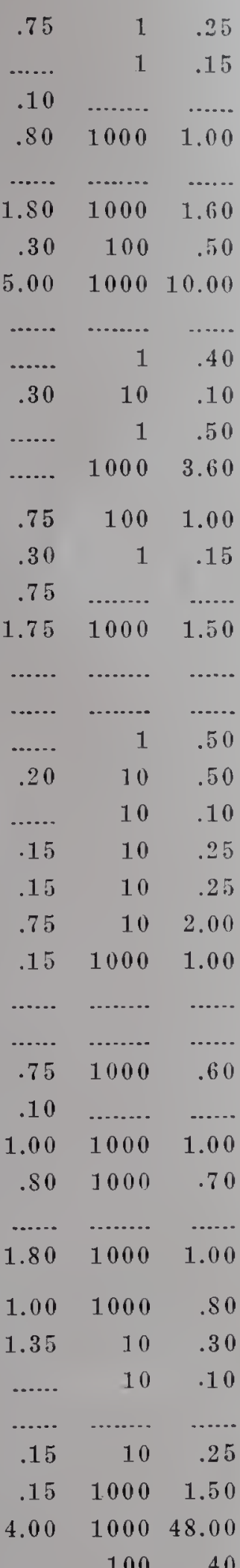

$\begin{array}{rr}.55 & .30\end{array}$

$9.00 \quad 5.00$

…. $\quad \cdots . .$.

…. . 30

2.50

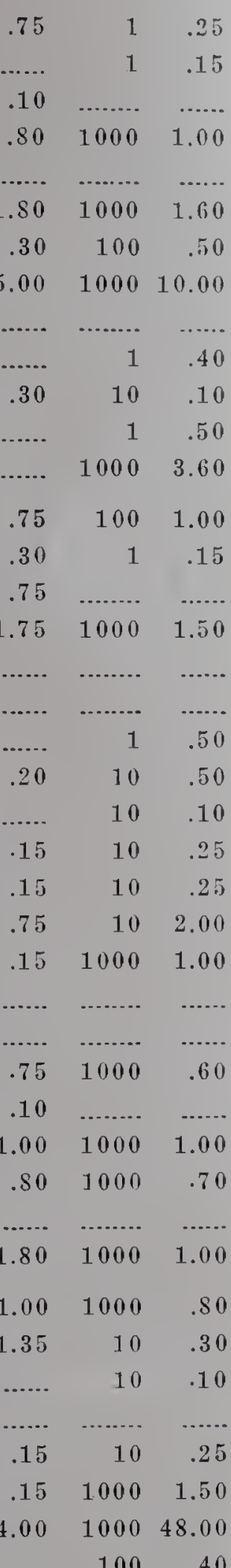

1.35

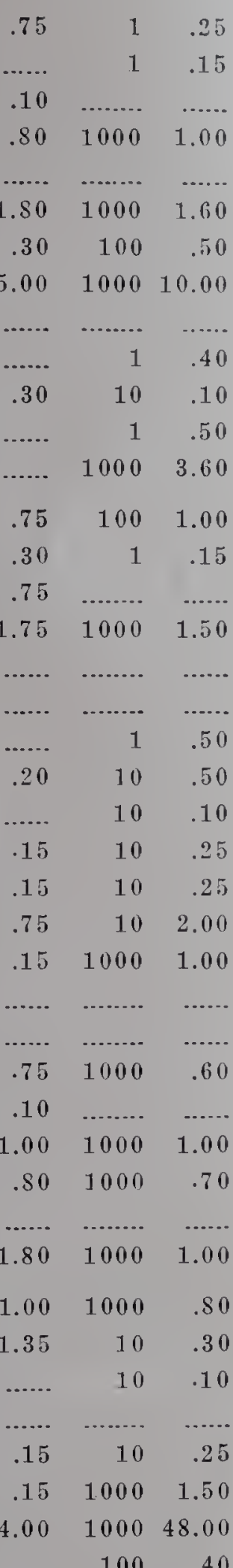

$.60 \quad .30$

..... .75

$3.50 \quad 1.75$

….. …...

2.50

…..

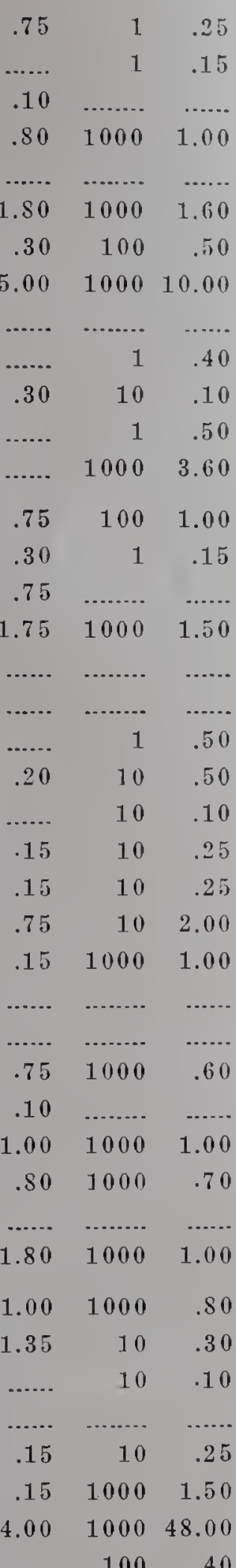

…..

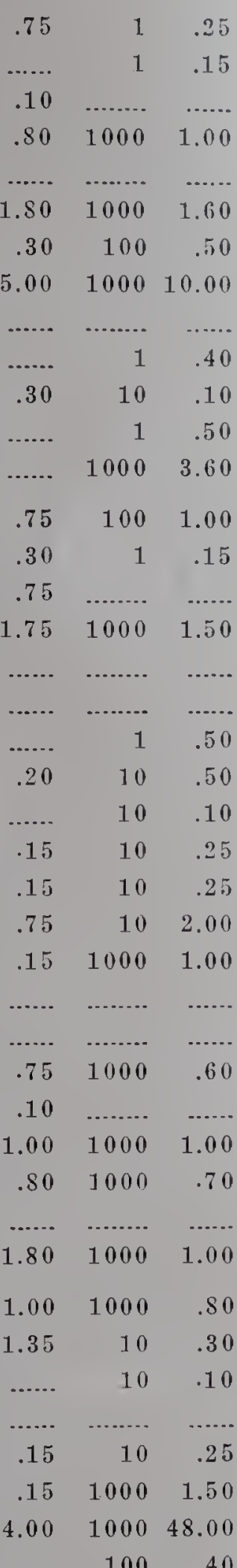

.25

1.25

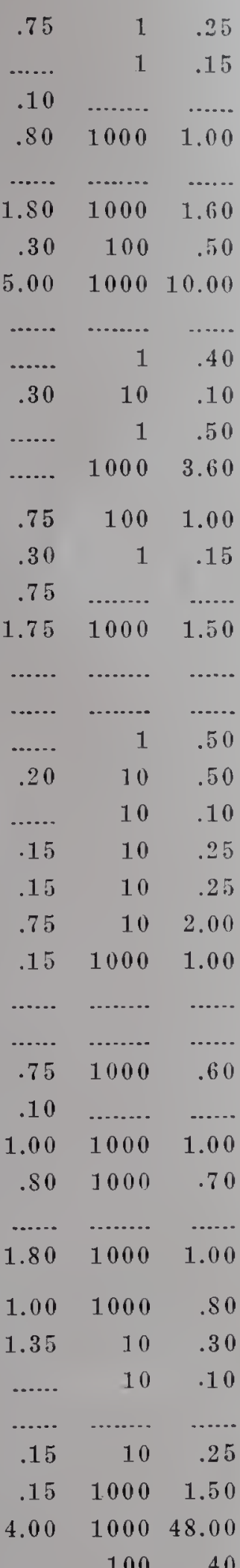

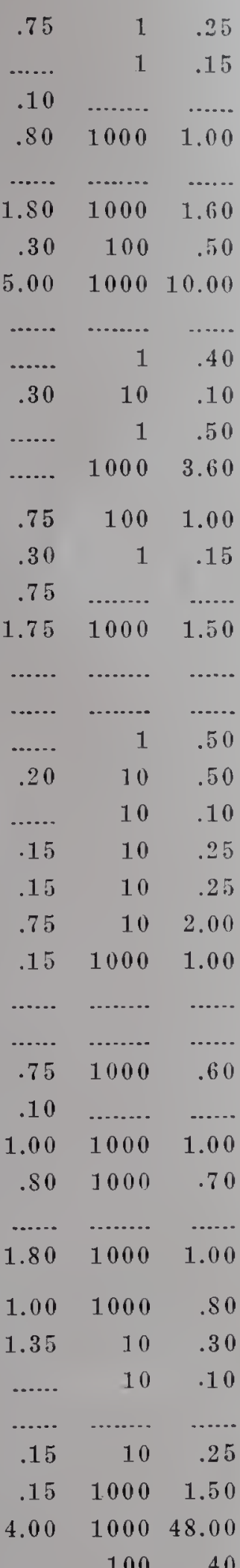

.30

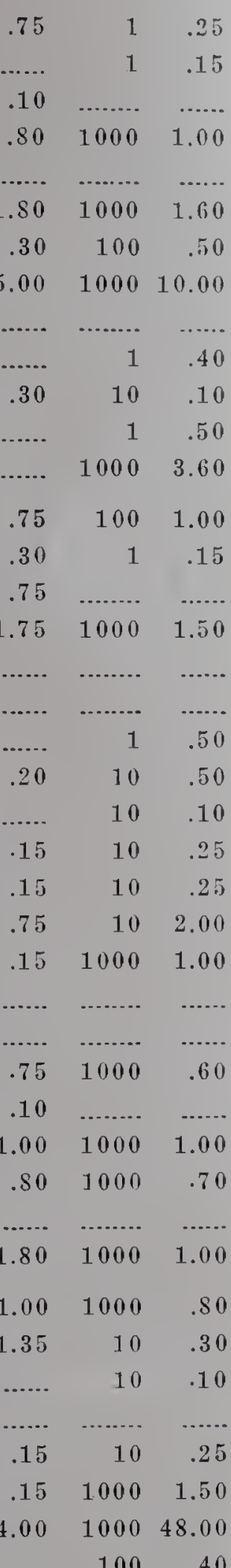

…...

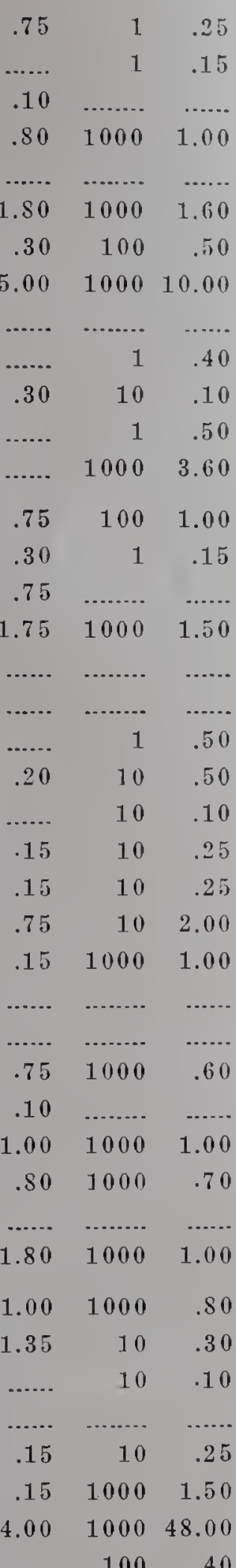

$1.50 \quad .75$

$.20 \quad .10$

2.00

1.00

1.60

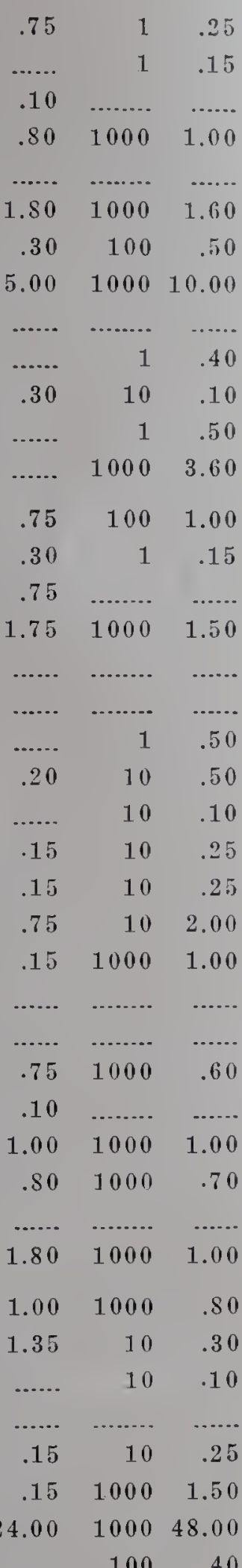

.30

3.60

4.00

2.40

4.50

.10

…..

1.00

1.35

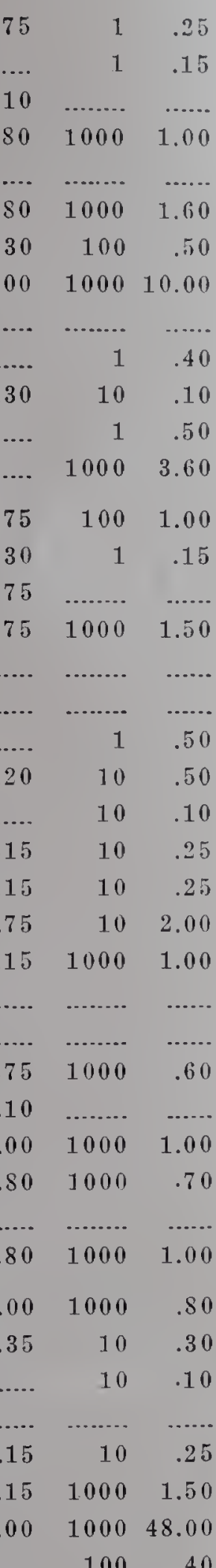

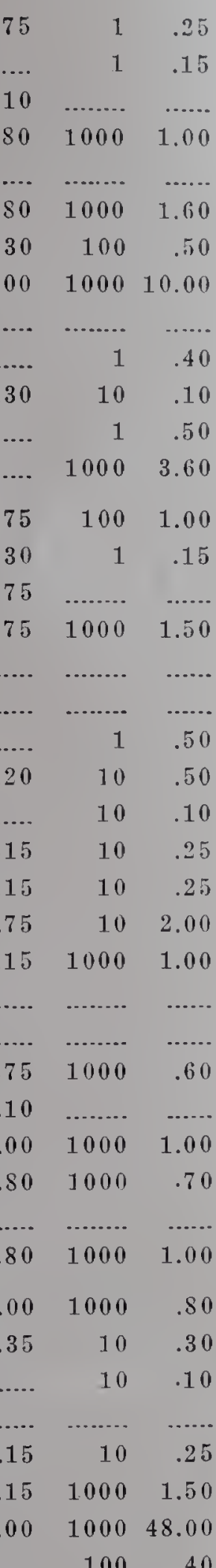

1.50

.35

......

.25

$.45 \quad .25$

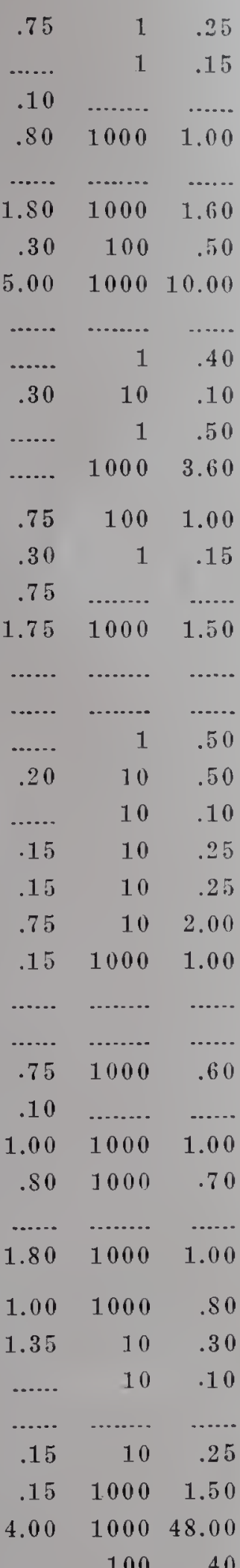

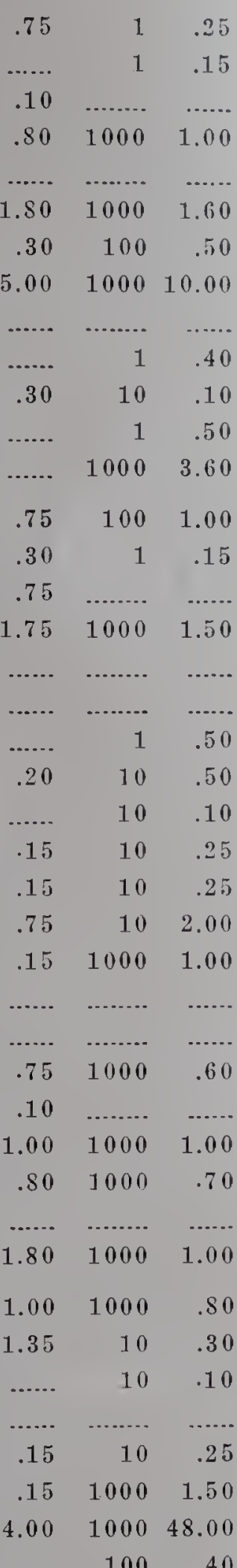

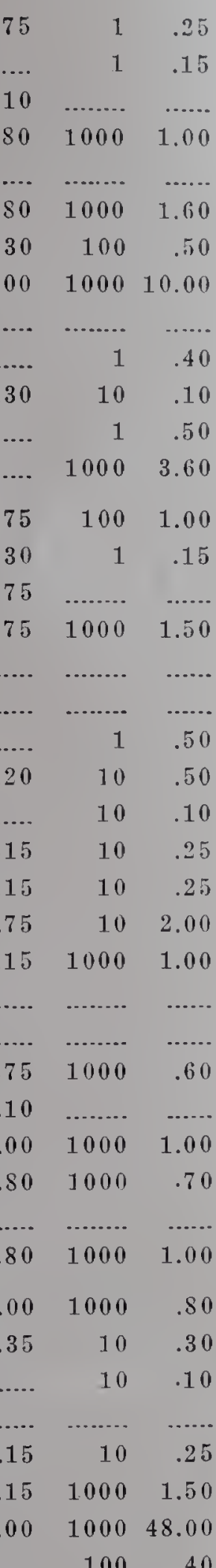

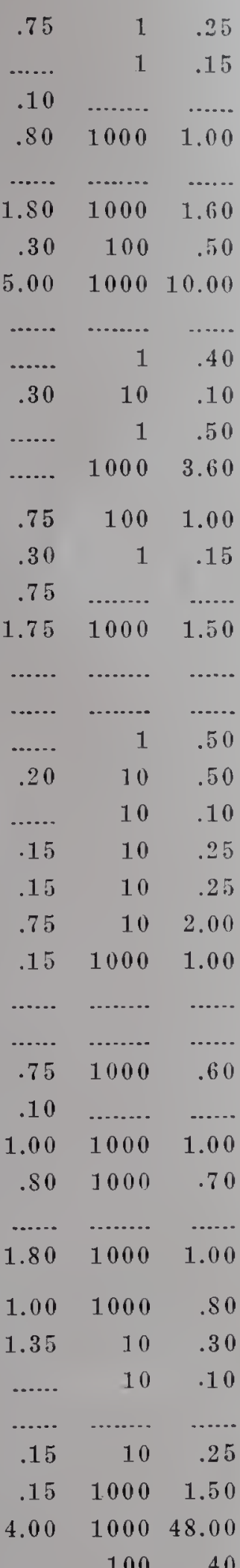

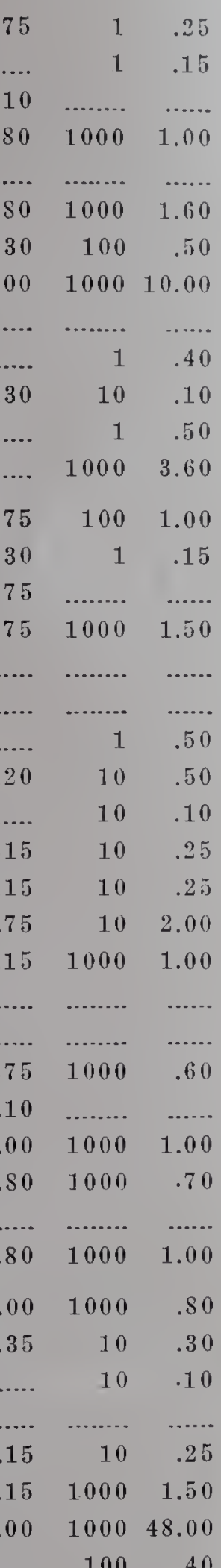

$\begin{array}{llllll}72.00 & 48.00 & 24.00 & 1000 & 48.00\end{array}$

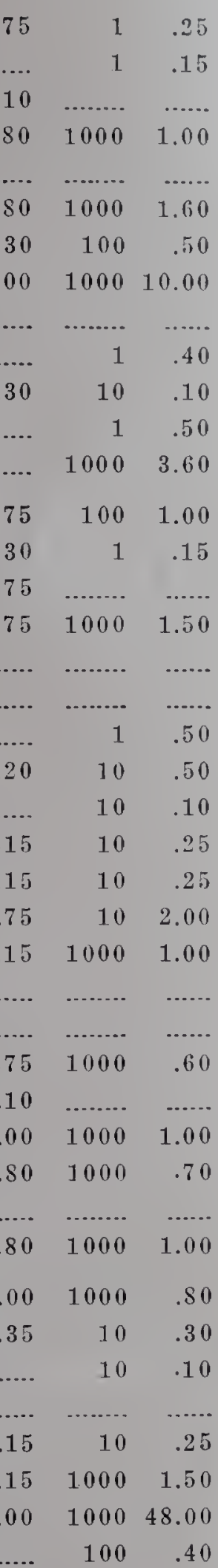

1.75

$1 \quad .35$

... ......

.25

$.25 \quad .15$

........

......

.50

3.60

4.50

5.60

......

10

.25

$10 \quad .20$

$\begin{array}{llll}2.25 & 1.35 & 100 & 2.00\end{array}$

$\begin{array}{lllll}.60 & 3.20 & 1.60 & 1000 & 1.50\end{array}$

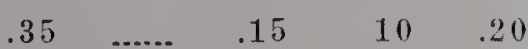

$\begin{array}{lllll}.90 & .45 & .25 & 100 & .25\end{array}$

$\begin{array}{lllll}3.50 & 2.00 & 1.00 & 1000 & 1.00\end{array}$

One at one-ninth 10 price; 10 at one-eighth 100 price; 100 at one-eighth 1000 price 


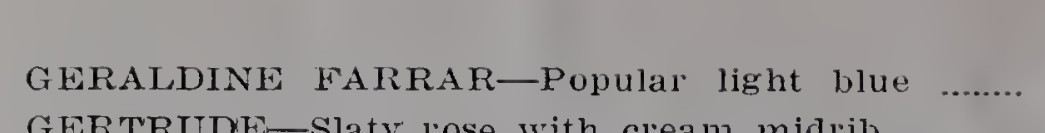

$\mathbf{P}$
100
1
1
1

Large Med. Small
Per 1 $1 / 4$ "up $3 / 4$ " up $3 / 8$ "up

Bulblets

(1/4 up $3 / 4$ " up $3 / 8$ "up Per Price

GERTRUDE-Slaty rose with cream midrib

$\begin{array}{lllll}3.60 & 2.00 & 1.00 & 1000 & 1.00\end{array}$

GERTRUDE ERREY-Shell pink, lighter center... 100

GERTRUDE GREY-New sparkling gray novelty

3.15

1.80

.90

$100 \quad 1.50$

G. J. MACKAY - Very ruffled salmon orange

-.....

…. $.80-1000 \quad .75$

GLADYS-Tall coral red, white throat

-......

.75

.50

.50

$1 \quad .20$

.75

GLORIOLA-Tall orange salmon, ruffled

.50

.30

$10 \quad .45$

.35

GLORIOSA-Bright light scarlet, white throat

.50

GOLDEN CHIMES-Fine new ruffled yellow

3.00

GOLDEN CUP-Very fine new deep yellow

2.00

GOLDEN DREAM-Very tall deep yellow

2.85

GOLD EAGLE-Good early commercial yellow

3.20

2.25

GOLDFINCH-Tall, very deep yellow

.50

GOVERNOR MEIER-Good new geranium pink ...

GRACE KIMBALT-Rare light violet blue

GRAF ZEPPELIN-Clear brilliant orange scarlet....

HAMPDEN-Salmon pink, rose purple throat .......

HEALESVILLE GLORY-TVine shade, darker throat

HEATHFIELD-Grenadine orange exhibition sort

HEA VENLY BLUE-Strong spikes of light blue ....

HELEN HOWARD-Golden buff. Coral markings 10

HELEN JACOBS-Fine pink and cream

HELIOSA-Very deep ruffled yellow. Rare

HERCULES-Clear buff, tall and massive

......

.25

10

10

.50

…...

1.40

1.00

.25

.40

.80

1.45

1.80

1.35

......

1.50

......

$0 \quad 13.50$

$00 \quad 7.00$

3.50

3.00

.80

18.00

HINEMIOA-Tery deep rose, cream blotches

$100 \quad 20.00$

HYACINTH-Light rose pink, cream throat

14.00

IMP. HENRY FORD-Very good deep purple ....... 11.50

JMP. QUEEN OF THE NIGHT-Very deep maroon $100 \quad 3.20$

INSPIRATION-Laciniated shrimp pink, sold throat $10 \quad 16.00$

IRIS PHILLIPS-Good new exhibition lavender ... $\quad 1 \quad 3.00$

ISABELLA-Salmon pink prim. grand.

$10 \quad 2.25$

IVANHOE-Clear light lilac, purple featler

.75

IVERNIA-Light rose pink, many out

$\cdots$

…...

1.50

9.00

3.50

2.00

1.80

.50

5.00

......

.75

.70

.75

......

.25

.50

1.00

4.50

1.50

1.00

.90

.30

12.00

8.00

100

2.50

JANE ADDAMS-Very lovely large lavender

JANET-Smoky gray, shaded deep pink

......

.70

$9.00 \quad 5.00$

5.00
1.50

1000

.15

......

1.50
.90

1.35

.50

.25

100

.80

$1 \quad .40$

$100 \quad 1.60$

$10 \quad .50$

$1 \quad .50$

JANICE-Distinct smoky rose lavender

$10 \quad .55$

.35

.20

.10

.15

......

.10

JEANETTE-Light yellow, penciled carmine

.75

JESSIF-Fine pure pink with white throat

2.25

1.35

.90

$2.50 \quad 1.25$

JOERG'S WVHITE-One of the largest whites ....... 100

5.00

.75

.40

JOHN RAMSEY-Strong growing deep salmon rose

$1 \quad 1.25$

JONKHEER VAN TETS-One of the best whites...

$100 \quad 25.00$

15.0010 .00

100

.......

JUBILEE-Very large lavender. Good grower

10016.00

$8.00 \quad 4.00$

10

.20

$10 \quad .50$

$100 \quad 1.25$

$1000 \quad 2.00$

$10 \quad 1.00$

$1000 \quad 20.00$

$1000 \quad 4.00$

KING ARTHUR-New ruffled lavender, lalge

$\begin{array}{rrrrrr}1 & 8.00 & 5.00 & 2.50 & 1 & .75 \\ 10 & 1.25 & .75 & .40 & 1000 & 2.00 \\ 10 & .75 & .45 & .25 & 1000 & 3.00 \\ 10 & .60 & .40 & .20 & 100 & .30 \\ 100 & 4.00 & 2.00 & 1.00 & 1000 & 1.00 \\ 1 & .40 & \ldots \ldots & .20 & \ldots \ldots \ldots & \ldots \ldots \\ 1 & .75 & \ldots \ldots . & \ldots \ldots & 10 & .50 \\ 1 & 1.25 & \ldots \ldots & .50 & 1 & .10 \\ 1 & .60 & .40 & .20 & 1 & .10 \\ 10 & 1.50 & .90 & .60 & 1000 & 3.00\end{array}$

KING GEORGE-Popular new red, white throat...

KIRCHHOFF'S NEW VIOLET-Good reddish purple

KIRCHHOFE'S VIOLET-Old but always in demand

IRIMHILDE-Early lavender, cream throat

KYIA-New deep rosy lavender

I,A CROSSE-Salmon red, light yellow throat

LA FRANCE-Fine pure pink. Strong growth

IAUY LORENE-Exquisite pink, yellow throat ...

LA PALOMA-Pure orange. Fine form and spike

$10 \quad 1.50$

One at one-ninth 10 price; 10 at one-eighth 100 price; 100 at one-eighth 1000 price 
IAVENDER BRIDE-Smoky lavender. Tall

LEANDER-Salmon rose, crimson purple throat ..

I.E HABITANT-Purple orercast golden brown. Fine

LEVIATHAN-Strong rose pink, penciled purple...

IIBELLE-Heliotrope blue. Fine color

LIGHTHOLSE-Outstanding orange, white throat

LILIOLA-Lily-shaped white, red blotch

LILYDALE-Fine new exhibition white

LOCHNAGER-Dark rose, flaked slate

LOELLA-An attractive worth-while sinok!

LOUIS HEMION-Orange salmon, yellow blotch

LUCIFER-Scarlet orange, purple blotch

LUTHER BURBANK-American Beauty rose

L. VON BEETHOVEN-Very tall new smoky

MAGNA BLANCA-Tall white, yellow throat

MAGNET-Tall strong-growing deep yellow

MAID OF ORLEANS-Fine white, creain throat ....

MAMMOTH WHITE-Best exhibition white

MAORILAND-Old rose, striped steel blue

MARMIORA-Large gray lavender. One of the best 10

MARNIA-Extra sood luminous orange

MAROCCO-Pfitzer's fine blackish red. Extra

MAROLEE-Rich rose pink. Large flowers

IIARY FRET-Early lavender resembling Peter's ...

MARY GARDEN-New white with purple throat...

MARY J. COOLE-Early light rose pink

IIARY LOCKWOOD-Pure pink, crimson feather ....

MARY MARIE-Light rose pink, striped rose ........

MART O'MINE-Very large lily-like white

MILKMAID-Giant milk white. Many open

MINUET-Most popular clear lavender ................. 10

100

MISS DES MOINES-Extra fine new lavender ....... 10

MISS F. E. WILLARD-New salmon orange self ....

MISS JOY-Very lovely tall light pink

MME. GEO. BOULAY - White, crimson bloteh .......

MME. MOUNET SULLY-TVhite, crimson blotches....

MONARCH OF THE GLEN-Large salmon, luffled..

MOONDARA-Deep salmon, crimson blotch

MOORISH KING-Outstanding large deep maroon....

MOTHER MACHREE-Famous lavender glay.

MOUNT HOOD-Very large ruffled white

MRS. F. C. HORNBERGER-Popular late white ... 100

MRS. F. E. BENNETT-White feathered violet ........

MRS. GALBRAITH-Large, flaked salmon pink ... 10

IRS. LEON DOUGLAS-Gigantic salmon rose ... 100

IIR.S. P. W. SISSON-Tall pink, large flowers ....... 100

MRS. RAY P. CHASE-Exhibition white, flecked...

IIRS. S. A. ERREY-Wonderful exhibition orange...

3.25

$1 \quad .75$

MRS. T. E. LANGFORD-New apple blossom pink...

IIRS. T. J. KNUDSON-Good medium violet blue ...

IIRS. TOM RATTRAY-Tall exhibition pink

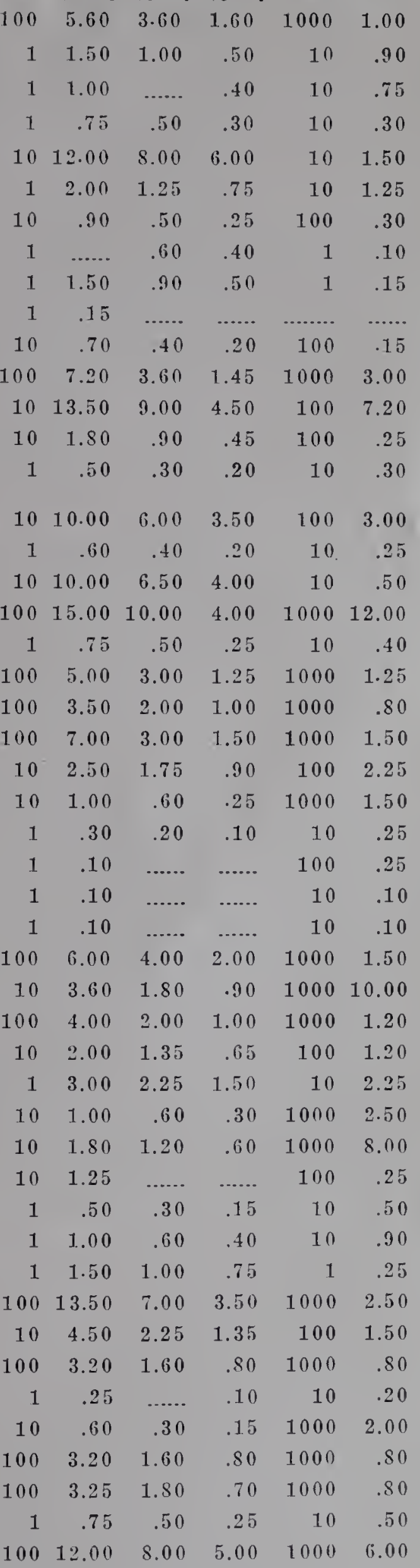

$1 \ldots 1.25$

$1 \quad 1.50 \quad 1.00$

1.50

MRS. VAN KONYNENBURG-Good light blue $100 \quad 4.00$
1.00

90

75

50

1.25

15

15

3.00

20

30

.00

25

.40

.80

1.50

2.25

1.50

.25

.25

.10

00

1.20

1.20

2.25

2.50

.00

25

50

90

.25

.50

1.50

80

.20

00

80

50

6.00

$10 \quad 1.00$

$\begin{array}{llll}2.00 & 1.00 & 1000 & .80\end{array}$

One at onc-ninth 10 price; 10 at one-eighth 100 price; 100 at one-eighth 1000 price 
MR. W. CUTHBERTSON-Clear ruffled salmon rose

NANCY HANKS-Rich apricot pink Good spike $100 \quad 3.20$

NELLY-Beautiful rose with white throat.

NERISSA-Salmon pink, edged blue, blotched ..

NEWINGTON-Exhibition white with yellow blotch

NOEL L. REEVE-Very fine exhibition rose pink ....

OLIVAR-Light blue veined darker

ORAFLAME-Deep yellow, shaded salmon

ORANGE WONDER-Fine self-colored orange .... 100

OREGON SUNSET-Very large pure clear yellow.... 10

ORIENTAL SILK-Rose pink veined darker ........ 100

ORIANDO-Grenadine red, purple feather, many out

OSALIN-Coral pink with orange throat

1013.50

OTHELLO-Violet blue with crimson feather

$100 \quad 3.50$

OUR SELECTION-Sinoky salmon. Many out

$10 \quad .60$

$10 \quad 1.60$

PAISLEY - Grayish eosine pink. Large and tall

10

PAUL CAMBON-Tall orange, maroon blotch ....

PAUL DESCHANEL-Wonderful rose with blotch.. 10

PAULINE KUNDERD-Rose pink, yellow throat....

PAUL PFITZER-Fine spike of velvety purple..

PEARL OF CALIFORNIA-Tall light pink

PELEGRINA-Outstanding new violet blue

............ 10

100

2.50

.35

2.70

2.70
60

3.50

3.50

16.00

$10 \quad 45.00 \quad 30.00$

PENINSULA BELLE-Amaryllis-like salmon pink..

PFITZER'S TRIUMPH-Mammoth orange salmon.. 10

PICARDY-Outstanding large shrimp pink

PIMPERNEL-Clear light red, white throat

PIRATE-Tall carmine red, darker throat

..............

............

PRIDE OF OREGON Deep pink Phipps .............

PRIDE OF PORTLAND-Tall, large, light coral red

PRIMATE-Good deep yellow prim. grand.

2.20

$\begin{array}{lll}00 & 3.50 & 2.00\end{array}$

$\begin{array}{lll}10 & 16.00 & 10.00\end{array}$

1.50

11.80

$\begin{array}{ll}00 & 12.00\end{array}$

7.20

$10 \quad 1.60$

$10 \quad 1.50$

PRIMROSE PRINCESS-Extra good light yellow .... 100

PSITTACINUS-Parrot species. Greenish shade ..... 10

PURPLE GLORY-Dark ruffled purple red

4.80

3.60

$100 \quad 3.50$

PURPLE VICTORY-Purplish crimson, large spike $1 \quad 1.00$

QUARTINTANUS-Species. Late bloomer. Red $\ldots \begin{array}{lll}\text {... } & 1 & .40\end{array}$

QUEEN MARY-Mair's large exhibition white ........ $1 \quad$.50

RAMESES-Strong growing, large exhibition purple $10 \quad \mathbf{1 3 . 5 0}$

RAPTURE-Tall light salmon rose, several out ........ $\quad \begin{array}{ll}1 & 1.00\end{array}$

RED GLORY - Crimson sport of Purple Glory

$\begin{array}{rr}1 & 1.00 \\ 10 & .50\end{array}$

RED LORY-Tall red with rose purple marking.

RED PHIPPS-Good new commercial red

$\begin{array}{llll}100 & 25.00 & 17.50 & 10.00\end{array}$

$10 \quad 7.00 \quad 3.80$

RED TORNADO-Rich scarlet, crimson blotch ...

RIDEAU-New orange salmon, several out

RIPPLING WATERS-Cream tinted with pink

RITA BECK-One of the finest pure pinks

$\mathrm{k}$

1

11.00

$1 \quad .15$

$100 \quad 4.50$

10020.00

12.00

ROSA BELLA-Pure pink, light feather

France ...

ROSELLA AMOS-New slaty purple, white throat....

ROSEMARIE PFITZER-Tinted exhibition white ....

ROSA RAISA-New dark violet blue

ROSEWOOD-Dark smoky rose pink
15.00

$1 \quad 2.50$

$10 \quad 1.25$
$1 \quad .50$
1.00

9.00

.80

1.00

2.40

1.80

2.00

.60

.20

......

4.50

100

6.40

Bulblets

$1000 \quad .80$

$\begin{array}{ll}10 & .40\end{array}$

$1000 \quad 48.00$

$10 \quad 1.35$

$1 \quad .25$

$100 \quad .15$

$\begin{array}{lll}.40 & 100 \quad .75\end{array}$

$\begin{array}{lll}1.50 & 1000 & 3.00\end{array}$

$\begin{array}{lll}1.50 & 100 & 2.50\end{array}$

$\begin{array}{lll}1.50 & 1000 & 2.00\end{array}$

$\begin{array}{lll}4.50 & 100 & 7.20\end{array}$

$\begin{array}{lll}1.00 & 1000 \quad .80\end{array}$

$\begin{array}{lll}.20 & 100 & .30\end{array}$

$.40 \quad 1000 \quad 4.80$

$\begin{array}{lll}.75 & 1000 & 4.00\end{array}$

$\begin{array}{lll}.15 & 10 & .15\end{array}$

$\begin{array}{lll}.90 & 100 & 2.00\end{array}$

$\begin{array}{lll}.25 & 1 & .10\end{array}$

$.80 \quad 1000 \quad 2.50$

$\begin{array}{lll}1.50 & 1000 \quad 2.00\end{array}$

$2.50 \quad 10 \quad 6.00$

$100 \quad 50.00$

.10

1.00

6.00

$1000 \quad 1.75$

$100 \quad 12.00$

.50

3.60

.40

.50

1.20

.90

1.00

.30

.10

$10 \quad 1.00$

$1000 \quad 4.00$

$1000 \quad 5.00$

$100 \quad 6.00$

$1000 \quad .80$

$100 \quad 2.00$

$1000 \quad .80$

$10 \quad .75$

$10 \quad .25$

.15

$100 \quad .15$

$1000 \quad 22.50$

$100 \quad 5.00$

$100 \quad 1.00$

.10

2.70

.......

.50

$1000 \quad 2.50$

1.50

$1000 \quad 10.00$

$\begin{array}{rrr}7.20 & 1000 & 10.00 \\ .75 & 10 & 1.80\end{array}$

$1.25 \quad .75$

$\begin{array}{rr}10 & 1.80 \\ 10 & .50\end{array}$

$3.00 \quad 1.50$

.75

.50

.25

$\begin{array}{lll}.40 & 100 & .40\end{array}$

One at one-ninth 10 price; 10 at one-eighth 100 price; 100 at one-eighth 1000 price 
ROYAL LAVENDER-Excellent rose la -endel ....

Large Med. Small

ROZAN-New rose pink. Immense size

Large Med. Small Bulblets
Per 1 $1 / 4$ " up $3 / 4$ "'up $3 / 8$ "'up Per Price

$100 \quad 6.00$

10

ST. BRUNO-Salmon pink, blushed chocolate

10

S'I. CECILIA-Rose pink with white throat.

SALBACH'S ORCHID-Fine new lavender rose ...

$\begin{array}{rr}1 & \cdots \\ 1 & .50\end{array}$

SALBACH'S PINK-Large rose pink, cream throat

1010.00

SCARLET WONDER-Very large clark red

1.50 $100 \quad 3.20$

SCHWABENGIRT-Pfitzer's fine new carly pink

SENORITA (L)-Distinct mahogany brown ...........

SEPTEMBER MORN-Tall strong orange pink ....

SKYLARK-Smoky gray from England

11.00

1.15

$0 \quad 4.50$

4.50

SMITING MIAESTRO-Very fine ruffled salmon .... 15.00

SOLVEIG-Good new ruffled white ........................ 140.00

SOUTHERN SKIES-Odd rose pink, rose and white $1 \quad .10$

$\begin{array}{llllll}\text { SPIRIT OF ST. LOUIS-Buff tinted orange red ... } & 10 & .70\end{array}$

SPRAY OF GOLD-New early deep yellow ........... 1 . 1 .65

STAR OF THE SEA-Peach red, marked brown.... $100 \quad 6.40$

STATELY LAVENDER-Light flecked lavender .... $\quad 1 \quad 1.00$

STUTTGARDIA-Extra fine glowing red .............. $10 \quad 2.00$

SUL'TAN-Crow's ruffled dark crimson

2.00

SULTAN-Mair's smoky salmon, cream blotch ....... $1 \quad$.50

SUN GOD-Rose pink, large cream blotch .......... 11.00

TAHWAKI-Fine new unfading orange, distinct ... $1 \quad 5.00$

THOS. A. EDISON-Rich clear dark red

.60

3.50

.30

1.50

1000

3.00

$\begin{array}{llll}.45 & .25 & 100 \quad .20\end{array}$

TIGER-Smoky sliade flaked salmon

100

TINAMBA-Clear pink, flaked salmon rose

TOBERSUN-New late yellow. Very good

10

......

.40

2.00

$\begin{array}{rrr}.50 & 1 & .10\end{array}$

-...........

$10 \quad .50$

$\begin{array}{llll}5.00 & 2.50 & 1.000 & 36.00\end{array}$

1.00

.75

$10 \quad .15$

.80

$.70 \quad 1000 \quad .60$

$\begin{array}{llll}.60 & .35 & 10 & .40\end{array}$

-......

…...

-......

......

1.35

1.35

10

.25

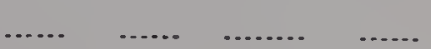

$\begin{array}{llll}\ldots . . . & \ldots . . & 1 & 4.00\end{array}$

.35

…..

$10 \quad .10$

.40

4.00

.20

$100 \quad .25$

$\begin{array}{lll}.20 & 10 & .40\end{array}$

$\begin{array}{lll}.00 & 1000 \quad 2.00\end{array}$

1.20

-.....

$10 \quad .75$

1.25

.70

$100 \quad 1.50$

$10 \quad 1.50$

TRIUMPHATOR-New tall orange red

3.00

......

1.20

$2.00 \quad 1.00$

10

$.60 \quad 1000$

11.00

TROUBADOUR-Very best red purple

$10 \quad 2.25$

-.....

.50

1.35

.75

TRULY BROWN-Popular purplish brown

$10 \quad 3.60$

1.80

$100 \quad 18.00 \quad 10.80$

.90

5.40

100

1.50

TUTENAKEI-Light scarlet, flaked black

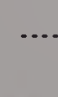

VALENCIA-Wonderful ruffled golden orange

$1 \quad 2.00$

VEILED BRILLIANCE-Pink tinted grayish blue .... 100

VICTOR-Brilliant scarlet, white blotch

3.60

-.....

......

100

1000

2.00

7.20

VEILCHENBLAU-Dark blue. Largc flower's ....... 100

WASAGA-New clear apricot, buff throat

6.00

3.00

1.00

$1 \ldots . . . . .$.

4.50

2.50

1.50

$1000 \quad 1.00$

$1000 \quad 2.00$

14.50

2.75

1.00

$1000 \quad 1.75$

WHITE PEARI_Fine pearly white. Many out ....... 10

W. H. PHIPPS-Mammoth spike salmon pink ....... 100

1.35

.80

11.00

WINGED VICTORY—Enormous salmon pink .......

WINGS OF THE MORNING-Early eosine pink ... 100

WODAN-Very dark blue, white rib

$10 \quad 2.00 \quad 1.00$

$.45-100 \quad .25$

$.80 \quad 1000 \quad .60$

$.50 \quad 1000 \quad 7.50$

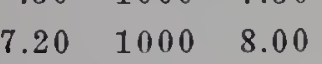

WOLFGANG VON GOETHE-Extra raspherry red

WUR'TEMBERGIA-Fine scarlet, creanı blotch ....

$0 \quad 28.80 \quad 14.40$

$\begin{array}{rrr}.30 & 1 & .10\end{array}$

$\begin{array}{llllll}10 & 11.25 & 6.50 & 3.60 & 10 & .50\end{array}$

$\begin{array}{llllll}10 & 18.00 & 8.00 & 5.00 & 100 & 8.00\end{array}$

YELLOW PERFECTION-Extra fine pure yellow.... $100 \quad 25.00 \quad 16.00 \quad 8.00 \quad 1000 \quad 12.00$ $\begin{array}{lllllllll}\text { YVONNE-Splendid flesh white, blotched red } \ldots \ldots \ldots & 10 & .60 & .30 & .15 & 1000 & 1.00\end{array}$

The above! list was compiled just before digging time. It is possible that in many cases varieties can be furnished in larger quantities than listed above. If interested in large lots, write us. We probably can supply them.

One at onc-ninth 10 price; 10 at one-eighth 100 price; 100 at one-eighth 1000 price 


\section{Real Bargain Collections}

Not over two collections of one kind will be sold to any customer. One each of the six collections on this page for \$12.50. Special discounts on page one do not apply to collections.

No. 1 - EUROPEAN - \$2

$1 \mathrm{~S}$ Blue Triumphator

1 L Commander Koehl

1 L Duchess of York

$1 \mathrm{~L}$ Krimhilde

1 L Mammoth White

1 L Marocco

1 L Roi Albert

$1 \mathrm{~L}$ Wuertembergia

1 L Yellow Perfection

No. 3 - AMERICAN - $\$ 2$

1 L Betty Nuthall

1 L Blue Moon

1 L Damascus

1 L Eugenie

1 L Geraldine Farrar

1 L Helen Jacobs

1 L Mother Machree

1 L Mount Hood

1 M Salbach's Orchid

No. 5 - RARE BULBLETS - \$2

Two of each:

A. H. Woodfull

Bagdad

Dora Gill

Eugenie

G. J. Mackay

Heathfield

Lucifer

Milkmaid

Pelegrina

Picardy

Rameses
No. 2-AUSTRALASIAN-\$2

$1 \mathrm{~L}$ Allawah

1 L Canberra

$1 \mathrm{~L}$ Hinemoa

1 I. Marmora

1 L Milkmaid

1 L Mrs. S. A. Errey

1 L Nerissa

1 L Red Lory

1 L Tutenakei

$1 \mathrm{~L}$ Victor

No. 4 - CUT FLOWER - $\$ 2$

10 large or 20 medium or 50 small of each of the following:

Dr. F. E. Bennett

Dr. Moody

Golden Dream

Mrs. F. C. Hornberger

Mrs. Leon Douglas

Mrs. P. W. Sisson

Nancy Hanks

W. H. Phipps

No. $6-$ EXHIBITION $-\$ 5$

One large bulb each:

Ave Maria

Bagdad

Blue Triumphator

Canberra

Commander Koehl

J. van Tets

Marmora

Picardy

Rameses

Wings of the Morning

We believe we have sufficient stock to fill all orders for abnve collections but reserve right to make substitution if stock of some variety is exhausted. 